\title{
Erratum: Efficient implementation of the $G W$ approximation within the all-electron FLAPW method [Phys. Rev. B 81, 125102 (2010)]
}

\author{
Christoph Friedrich $\odot$, Stefan Blügel, and Arno Schindlmayr
}

(Received 17 May 2021; published 8 July 2021)

DOI: 10.1103/PhysRevB.104.039901

Figure 1 is a correction of Fig. 6 of the original paper. The (unchanged) caption defines the dashed and solid lines as localspin-density approximation (LSDA) and $G W$ quasiparticle bands, respectively, whereas these lines were erroneously reversed in the actual figure. We have corrected the figure in Fig. 1. The explanation and conclusions in the main text of the original paper are not affected and remain unchanged.

The second matrix in Eq. (36) of the original paper is incorrect. The corrected formula reads

$$
\begin{aligned}
W_{\mu \nu}(\mathbf{k}, i \omega) \sim & \left(\begin{array}{cccc}
0 & 0 & \cdots & 0 \\
0 & \tilde{W}_{22}(\omega) & \cdots & \tilde{W}_{2 n}(\omega) \\
\vdots & \vdots & \ddots & \vdots \\
0 & \tilde{W}_{n 2}(\omega) & \cdots & \tilde{W}_{n n}(\omega)
\end{array}\right) \\
& +\frac{1}{\mathbf{e}_{\mathbf{k}}^{\mathrm{T}} \mathbf{L}(\omega) \mathbf{e}_{\mathbf{k}}}\left(\begin{array}{cccc}
4 \pi / k^{2} & \mathbf{e}_{\mathbf{k}}^{\mathrm{T}} \tilde{\mathbf{w}}_{2}(\omega) \sqrt{4 \pi} / k & \cdots & \mathbf{e}_{\mathbf{k}}^{\mathrm{T}} \tilde{\mathbf{w}}_{n}(\omega) \sqrt{4 \pi} / k \\
\mathbf{e}_{\mathbf{k}}^{\mathrm{T}} \tilde{\mathbf{w}}_{2}^{*}(\omega) \sqrt{4 \pi} / k & {\left[\mathbf{e}_{\mathbf{k}}^{\mathrm{T}} \tilde{\mathbf{w}}_{2}^{*}(\omega)\right]\left[\mathbf{e}_{\mathbf{k}}^{\mathrm{T}} \tilde{\mathbf{w}}_{2}(\omega)\right]} & \cdots & {\left[\mathbf{e}_{\mathbf{k}}^{\mathrm{T}} \tilde{\mathbf{w}}_{2}^{*}(\omega)\right]\left[\mathbf{e}_{\mathbf{k}}^{\mathrm{T}} \tilde{\mathbf{w}}_{n}(\omega)\right]} \\
\vdots & \vdots & \ddots & \vdots \\
\mathbf{e}_{\mathbf{k}}^{\mathrm{T}} \tilde{\mathbf{w}}_{n}^{*}(\omega) \sqrt{4 \pi} / k & {\left[\mathbf{e}_{\mathbf{k}}^{\mathrm{T}} \tilde{\mathbf{w}}_{n}^{*}(\omega)\right]\left[\mathbf{e}_{\mathbf{k}}^{\mathrm{T}} \tilde{\mathbf{w}}_{2}(\omega)\right]} & \cdots & {\left[\mathbf{e}_{\mathbf{k}}^{\mathrm{T}} \tilde{\mathbf{w}}_{n}^{*}(\omega)\right]\left[\mathbf{e}_{\mathbf{k}}^{\mathrm{T}} \tilde{\mathbf{w}}_{n}(\omega)\right]}
\end{array}\right),
\end{aligned}
$$

where we have introduced the scaled vectors $\tilde{\mathbf{w}}_{\mu}(\omega)=\sqrt{v_{\mu}(\mathbf{0})} \mathbf{w}_{\mu}(\omega)(\mu \geqslant 2)$ to simplify the notation. $\mathbf{w}_{\mu}(\omega)$ are the respective vectors appearing in the dielectric matrix, e.g., its wing elements are $\mathbf{e}_{\mathbf{k}}^{\mathrm{T}} \mathbf{w}_{\mu}(\omega) /\left[\mathbf{e}_{\mathbf{k}}^{\mathrm{T}} \mathbf{L}(\omega) \mathbf{e}_{\mathbf{k}}\right]$. Equations (39) and (40) of the original paper contain minor notational mistakes. In the former, the matrix indices are flipped,

$$
\mathbf{w}_{\mu}(\omega)=\sqrt{4 \pi} \sum_{\nu \geqslant 2} \tilde{\varepsilon}_{v \mu}^{-1}(\omega) \mathbf{s}_{\nu}(\omega) \sqrt{v_{\nu}(\mathbf{0})} .
$$

In the latter, a complex conjugation is missing,

$$
\mathbf{L}(\omega)=\mathbf{1}-4 \pi \mathbf{H}(\omega)-\sqrt{4 \pi} \sum_{\mu \geqslant 2} \mathbf{s}_{\mu}^{*}(\omega) \mathbf{w}_{\mu}^{\mathrm{T}}(\omega) \sqrt{v_{\mu}(\mathbf{0})} .
$$

The implemented formulas were and are correct. All of the text and conclusions of the original paper remain unaffected.

The generalization to arbitrary complex frequencies $(i \omega \rightarrow \omega)$, including real frequencies, would make the matrices nonHermitian (complex). In particular, the elements in the first column would no longer be the complex conjugates of the ones in the first row. However, the above formulas can still be applied if the starred and unstarred quantities [e.g., $\mathbf{w}_{\mu}^{*}(\omega)$ and $\mathbf{w}_{\mu}(\omega)$ ] are not interpreted as the complex conjugates of each other but as two independent quantities. Equation (2) would have to be complemented by $\mathbf{w}_{\mu}^{*}(\omega)=\sqrt{4 \pi} \sum_{\nu \geqslant 2} \tilde{\varepsilon}_{\mu \nu}^{-1}(\omega) \mathbf{s}_{v}^{*}(\omega) \sqrt{v_{v}(\mathbf{0})}$.

Finally, the imaginary part of the Drude formula in Eq. (41) of the original paper is not quite accurate. However, its qualitative form is correct: The imaginary part is nonzero only in direct proximity of $\omega=0$ (see the derivation below). For the actual implementation, the imaginary part does not play a role because the $1 / \omega^{2}$ singularity pushes the inverse dielectric function (with a factor $w^{2}$ ) to zero at $\omega=0$ and $\mathbf{k}=\mathbf{0}$. For all practical purposes, it thus suffices to replace Eq. (41) by

$$
P_{11}^{\mathrm{D}}(\mathbf{k}, i \omega) \sim-\frac{k^{2}}{4 \pi} \frac{\omega_{\mathrm{pl}}^{2}}{\omega^{2}} .
$$

Equation (42) of the original paper remains correct. We note that the frequency argument contains the imaginary unit. Without it, the sign would obviously be reversed, $P_{11}^{\mathrm{D}}(\mathbf{k}, \omega) \sim \frac{k^{2}}{4 \pi} \omega_{\mathrm{pl}}^{2} / \omega^{2}$.

For the sake of completeness, we want to give a derivation of the Drude formula because the work cited as Ref. [1] (Ref. [35] in the original paper) is hard to find and, to our knowledge, only available in German. Furthermore, we will consider the 


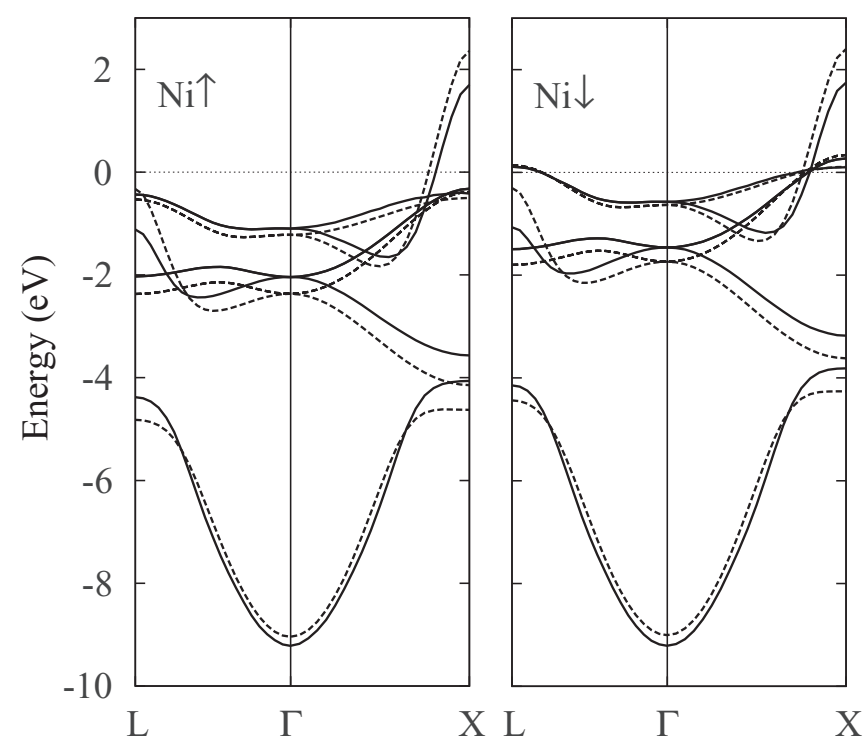

FIG. 1. Band structure of Ni calculated by the LSDA (dashed lines) and the $G W$ approximation (solid lines) for majority (left panel) and minority spin (right panel). The Fermi energy is set to zero. (Note that in the original paper the GW and LSDA bands were erroneously shown as dashed and solid lines, respectively, in contradiction to the caption.)

time-ordered response function, whereas the derivation in Ref. [1] is for the retarded response function. We will also show that the plasma frequency $\omega_{\mathrm{pl}}$ can be calculated from an integration over the Fermi surface.

In a pure plane-wave representation, the head $\left[P_{\mathbf{0 0}}(\mathbf{k}, \omega)\right]$ and wings $\left[P_{\mathbf{G} \mathbf{0}}(\mathbf{k}, \omega)\right.$ and $\left.P_{\mathbf{0 G}}(\mathbf{k}, \omega), \mathbf{G} \neq \mathbf{0}\right]$ of the polarization matrix obey a $k^{2}$ and $k$ dependence around the $\Gamma$ point, respectively, as shown in Eq. (35) of the original paper. The body elements $\left[P_{\mathbf{G G}^{\prime}}(\mathbf{k}, \omega), \mathbf{G} \neq \mathbf{0}, \mathbf{G}^{\prime} \neq \mathbf{0}\right]$ are finite at $\Gamma$. All this follows from Eq. (22) of the original paper and, for $n \neq n^{\prime}$, from $\left\langle e^{i \mathbf{k} \cdot \mathbf{r}} \varphi_{n \mathbf{q}}^{\sigma} \mid \varphi_{n^{\prime} \mathbf{q}+\mathbf{k}}^{\sigma}\right\rangle \propto k$ for $k \rightarrow 0$. For metals, however, the two band indices $n$ and $n^{\prime}$ can be identical, which gives rise to intraband terms (Drude term). The Drude term leaves the form of the polarization function for $k \rightarrow 0$ [Eq. (35)] unchanged and is a qualitatively and often quantitatively important contribution. In particular, it makes the static screened interaction fall off exponentially in metals, which guarantees an exact cancellation of the logarithmic divergence of (Hartree-Fock) band gradients (originating from the exchange self-energy). Without this cancellation, the bands would exhibit unphysical dispersion anomalies where they cross the Fermi surface.

In order to derive the Drude term, we rewrite Eq. (22) of the original paper in a pure plane-wave representation as

$$
P_{\mathbf{G G}^{\prime}}(\mathbf{k}, \omega)=\int_{-\infty}^{\infty} \frac{S_{\mathbf{G G}^{\prime}}\left(\mathbf{k}, \omega^{\prime}\right)}{\omega-\omega^{\prime}+i \eta \operatorname{sgn}\left(\omega^{\prime}\right)} d \omega^{\prime}
$$

with the spectral function

$$
S_{\mathbf{G G}^{\prime}}(\mathbf{k}, \omega)=\frac{1}{V} \sum_{\sigma} \sum_{\mathbf{q}}^{\mathrm{BZ}} \sum_{n}^{\text {occ unocc }} \sum_{n^{\prime}}\left\langle e^{i(\mathbf{k}+\mathbf{G}) \cdot \mathbf{r}} \varphi_{n \mathbf{q}}^{\sigma} \mid \varphi_{n^{\prime} \mathbf{q}+\mathbf{k}}^{\sigma}\right\rangle\left\langle\varphi_{n^{\prime} \mathbf{q}+\mathbf{k}}^{\sigma} \mid \varphi_{n \mathbf{q}}^{\sigma} e^{i\left(\mathbf{k}+\mathbf{G}^{\prime}\right) \cdot \mathbf{r}}\right\rangle\left[\delta\left(\omega+\epsilon_{n \mathbf{q}}^{\sigma}-\epsilon_{n^{\prime} \mathbf{q}+\mathbf{k}}^{\sigma}\right)-\delta\left(\omega-\epsilon_{n \mathbf{q}}^{\sigma}+\epsilon_{n^{\prime} \mathbf{q}+\mathbf{k}}^{\sigma}\right)\right] .
$$

The $n^{\prime}$ summation can be extended to all states $\left(\sum_{n^{\prime}}^{\text {unocc }} \rightarrow \sum_{n^{\prime}}^{\text {all }}\right)$ because the terms where both $n$ and $n^{\prime}$ states are occupied cancel pairwise. To see this, it is important to note that with $\varphi_{n \mathbf{q}}^{\sigma}(\mathbf{r})$ its complex conjugate $\varphi_{n \mathbf{q}}^{\sigma *}(\mathbf{r})$ is also an eigenfunction with the same eigenvalue. This step is helpful in the following because it saves us the need to expand the Fermi surface (as the dividing surface between filled and empty states) in $\mathbf{k}$.

We now consider the intraband terms with $n=n^{\prime}$ in the limit $k \rightarrow 0$. Expanding the expression in the square brackets gives

$$
\begin{aligned}
\delta\left(\omega+\epsilon_{n \mathbf{q}}^{\sigma}-\epsilon_{n \mathbf{q}+\mathbf{k}}^{\sigma}\right)-\delta\left(\omega-\epsilon_{n \mathbf{q}}^{\sigma}+\epsilon_{n \mathbf{q}+\mathbf{k}}^{\sigma}\right) & =2\left(\epsilon_{n \mathbf{q}}^{\sigma}-\epsilon_{n \mathbf{q}+\mathbf{k}}^{\sigma}\right) \frac{d}{d \omega} \delta(\omega)+O\left[\left(\epsilon_{n \mathbf{q}}^{\sigma}-\epsilon_{n \mathbf{q}+\mathbf{k}}^{\sigma}\right)^{3}\right] \\
& =-\left[2\left(\mathbf{k}^{\mathrm{T}} \nabla_{\mathbf{q}}\right) \epsilon_{n \mathbf{q}}^{\sigma}+\left(\mathbf{k}^{\mathrm{T}} \nabla_{\mathbf{q}}\right)^{2} \epsilon_{n \mathbf{q}}^{\sigma}\right] \frac{d}{d \omega} \delta(\omega)+O\left(k^{3}\right) .
\end{aligned}
$$

As the intraband contribution to Eq. (6) is thus at least of linear order in $k$ (the $\langle\cdots\rangle$ are finite), there are no relevant contributions to the body elements of the polarization matrix. There are no relevant contributions to the wing elements either because the term linear in $k$ vanishes when summed (or integrated) over $\mathbf{q}\left(\nabla_{\mathbf{q}} \epsilon_{n,-\mathbf{q}}^{\sigma}=-\nabla_{\mathbf{q}} \epsilon_{n \mathbf{q}}^{\sigma}\right.$ and $\left.\left.\nabla_{\mathbf{q}} \epsilon_{n \mathbf{q}}^{\sigma}\right|_{q=0}=0\right)$. As a result, we only obtain a 
contribution (of relevant order) for the head element

$$
S_{\mathbf{0 0}}(\mathbf{k}, \omega) \stackrel{k \rightarrow 0}{\sim}-\frac{k^{2}}{4 \pi}\left\{\frac{4 \pi}{V} \sum_{\sigma} \sum_{\mathbf{q}}^{\mathrm{BZ}} \sum_{n}^{\mathrm{occ}}\left[\left(\mathbf{e}_{\mathbf{k}}^{\mathrm{T}} \nabla_{\mathbf{q}}\right)^{2} \epsilon_{n \mathbf{q}}^{\sigma}\right]\right\} \frac{d}{d \omega} \delta(\omega)=-\frac{k^{2} \omega_{\mathrm{pl}}^{2}}{4 \pi} \frac{d}{d \omega} \delta(\omega)
$$

with the plasma frequency $\omega_{\mathrm{pl}}=\omega_{\mathrm{pl}}\left(\mathbf{e}_{\mathbf{k}}\right)$. We have used that $\left\langle e^{i \mathbf{k} \cdot \mathbf{r}} \varphi_{n \mathbf{q}}^{\sigma} \mid \varphi_{n^{\prime} \mathbf{q}+\mathbf{k}}^{\sigma}\right\rangle \rightarrow 1$ for $k \rightarrow 0$ and $n=n^{\prime}$. Through Eq. (5), we finally obtain

$$
P_{\mathbf{0 0}}(\mathbf{k}, \omega) \stackrel{k \rightarrow 0}{\sim}-\frac{k^{2} \omega_{\mathrm{pl}}^{2}}{4 \pi} \int_{-\infty}^{\infty} \frac{\frac{d}{d \omega^{\prime}} \delta\left(\omega^{\prime}\right)}{\omega-\omega^{\prime}+\operatorname{sgn}\left(\omega^{\prime}\right) i \eta} d \omega^{\prime}=\frac{k^{2} \omega_{\mathrm{pl}}^{2}}{4 \pi}\left(\frac{1}{\omega^{2}}-i \pi\left|\frac{d}{d \omega} \delta(\omega)\right|\right) .
$$

The imaginary part can be pictured as two (scaled) delta peaks, symmetrical around $\omega=0$ and infinitely close to each other, $|d \delta(\omega) / d \omega|=\lim _{\eta \rightarrow 0^{+}}[\delta(\omega+\eta)+\delta(\omega-\eta)] /(2 \eta)$. The Drude contribution to the dielectric function is $-\omega_{\mathrm{pl}}^{2}\left(1 / \omega^{2}-\right.$ $i \pi|d \delta(\omega) / d \omega|$ ). The difference from the formula given in the original paper (apart from the sign $i^{2}=-1$ ) is in the imaginary component, which proves irrelevant for the implementation, however, as already pointed out.

In general, $\omega_{\mathrm{p}}^{2}$ is a tensorial quantity of the type $\mathbf{e}_{\mathbf{k}}^{\mathrm{T}} \omega_{\mathrm{pl}}^{2} \mathbf{e}_{\mathbf{k}}$. In cubic systems, it becomes a scalar, which can be calculated efficiently by an integration over the Fermi surface,

$$
\omega_{\mathrm{pl}}^{2}=\frac{4 \pi}{3 V} \sum_{\sigma} \sum_{\mathbf{q}}^{\mathrm{BZ}} \sum_{n}^{\mathrm{occ}} \nabla_{\mathbf{q}}^{2} \epsilon_{n \mathbf{q}}^{\sigma}=\frac{4 \pi}{3 V} \sum_{\sigma} \sum_{n} \frac{V}{8 \pi^{3}} \int_{\epsilon_{n \mathbf{q}}^{\sigma} \leqslant \epsilon_{\mathrm{F}}} d^{3} q \nabla_{\mathbf{q}}^{2} \epsilon_{n \mathbf{q}}^{\sigma}=\frac{1}{6 \pi^{2}} \sum_{\sigma} \sum_{n} \int d^{3} q\left|\nabla_{\mathbf{q}} \epsilon_{n \mathbf{q}}^{\sigma}\right|^{2} \delta\left(\epsilon_{n \mathbf{q}}^{\sigma}-\epsilon_{\mathrm{F}}\right),
$$

where we have used the divergence theorem in the last step. The band gradient can be expressed as the expectation value of the momentum operator $\nabla_{\mathbf{q}} \epsilon_{n \mathbf{q}}^{\sigma}=\left\langle\varphi_{n \mathbf{q}}^{\sigma}|-i \nabla| \varphi_{n \mathbf{q}}^{\sigma}\right\rangle$. We employ the tetrahedron method to represent the Fermi surface in reciprocal space as a triangle mesh, on which the integration is carried out.

We are indebted to Walter Lambrecht and Jörn Stöhler who pointed out the notational inconsistency and error in Eq. (36) of the original paper.

[1] M. Taut, Meßgrößen und Bandstruktur, in Ergebnisse in der Elektronentheorie für Metalle, edited by P. Ziesche and G. Lehmann (Akademie/Springer, Berlin, 1983). 